\title{
Application of Incident Chain Model and Targeted Dissemination Technology in Early Warning System
}

\author{
Zhengqing Zhou' ${ }^{1}$, Jianguo Chen ${ }^{1}$, Peng Du${ }^{2}$, Zhanhui Sun ${ }^{1}$, Handong $\mathrm{Wu}^{1}{ }^{1}$, Hongyong Yuan${ }^{1}$ \\ ${ }^{1}$ Institute of Public Safety Research, Department of Engineering Physics, Tsinghua University, Beijing, China \\ ${ }^{2}$ Collaborative Innovation Center for Public Safety, Tsinghua University, Beijing GS Technology Co., Ltd., Beijing, China \\ Email: zhouzheng_qing@163.com, chenjianguo@tsinghua.edu.cn,pdu@tsinghua.org.cn, zhsun@tsinghua.edu.cn, \\ wuhandong@mail.tsinghua.edu.cn,hy-yuan@tsinghua.edu.cn
}

How to cite this paper: Zhou, Z.Q., Chen, J.G., Du, P., Sun, Z.H., Wu, H.D. and Yuan, H.Y. (2019) Application of Incident Chain Model and Targeted Dissemination Technology in Early Warning System. World Journal of Engineering and Technology, 7, 91-96.

https://doi.org/10.4236/wjet.2019.72B011

Received: May 10, 2019

Accepted: May 28, 2019

Published: May 31, 2019

\begin{abstract}
In order to improve the accuracy and efficiency of early warning system, the incident chain model and the targeted dissemination technology are proposed in this paper. Firstly, the occurrence probability, affected area and duration of disaster are predicted with the incident chain model and GIS. According to prediction results, the early warning system can accurately deliver early warning information specifically to the affected areas through targeted dissemination. Moreover, dissemination performance can also be evaluated in real time after early warning information dissemination, so that everyone in the affected area can receive early warning information successfully. The incident chain model and the targeted dissemination technology presented in this study are of great significance for improving the information dissemination ability of early warning system.
\end{abstract}

\section{Keywords}

Early Warning, Targeted Dissemination, Incident Chain Model, GIS

\section{Introduction}

According to Sendai Framework for Disaster Risk Reduction, over 700 thousand people have lost their lives, over 1.4 million have been injured and approximately 23 million have been made homeless as a result of disasters from 2005 to 2014 [1]. Timely and accurate dissemination for early warning information prior to disasters is of great significance for reducing the loss of lives and property. At present, most countries in the world have established early warning system [2] [3] [4] [5], and those systems have played a very important role in the emergen- 
cy management and response. However, the current early warning system can be further improved in a number of aspects. For example, the existing early warning systems tend to lack flexibly in defining dissemination areas. And as areas affected by disasters are usually not aligned with administrative divisions, people in the non-affected areas may also receive early warning information, which could lead to confusions, waste of system resources and ultimately affect the performance of the early warning services; Another potential issue is that authorities responsible for early warning usually have limited means to gauge the result of dissemination and whether people in the affected area have successfully received the warning.

In order to solve these problems, in this paper we propose to introduce incident chain model and targeted dissemination technology to the early warning system. This improved early warning system was implemented for Guangxi Province, China, and the resulting service statistics demonstrate notable performance enhancement.

\section{Risk Analysis Based on Incident Chain Model}

Disasters, whether natural or man-made, usually tend to cause one or more secondary disasters, a phenomenon referred to as the incident chain [6]. For example, rainstorms may cause floods, and floods could lead to landslides, debris flows, building collapses and other secondary disasters. In order to predict both primary and secondary disasters at the same time, an incident chain model for rainstorms is developed. According to incident chain model for rainstorms, 3D GIS data in specific areas and meteorological forecast information, the occurrence probability, development trend, affected area and duration of the flood caused by the rainstorms can be predicted. And then the flood predicted results will be used as the initial conditions for the calculation of other secondary disasters such as landslides, debris flows, building collapses and so on, i.e. the prediction results of the secondary disasters in the previous stage are taken as the initial conditions of the secondary disasters in the next stage. In this way, iterative calculation is carried out to predict all possible secondary disasters and their effects. Disaster impact prediction based on incident chain model is shown in Figure 1.

In summary, the incident chain model can accurately predict the disaster process, types and likelihood of secondary disasters, affected areas and impact. The prediction results provide a scientific basis for formulating early warning dissemination strategy, in other words, when a disaster occurs, the decision maker will be very clear about what type of early warning should be issued, when and where to issue this early warning information.

\section{Targeted Dissemination Technology in Early Warning System}

According to prediction results based on incident chain model in Section 2, targeted dissemination technology can recommend appropriate channels to quickly 




Figure 1. Disaster impact prediction based on incident chain model.

and accurately deliver early warning information specifically to the affected areas, so targeted dissemination technology is an important technology in improving the early warning system.

The targeted dissemination technology proposed in this paper allows for the flexible definition for early warning area, the terminal performance monitoring in the early warning areas and the performance evaluation after early warning information dissemination. The details are as follows:

1) Flexible definition for early warning area

The affected area is determined based on predictions generated with the incident chain model. Then the early warning system can accordingly define the early warning area in a flexible manner. The dissemination area may be defined using various levels administrative divisions, or simply by plotting of polygons, circles, or any user-defined shapes. In addition, the system also supports the dissemination of early warning information to entities such as roads and railways, etc. Some types of the early warning area are shown in Figure 2.

As a result, this early warning system can specifically restrict the delivery of given early warning information to user-defined areas, which enables fine-tuned dissemination of multiple warning messages to different or even adjacent areas whilst ensures other areas will not receive unintended warnings.

2) Terminal monitoring in the early warning areas

After the early warning area is determined, the system can automatically identify all dissemination terminals in the early warning areas. At the same time, the information such as the operating status, location, and quantity of the dissemination terminals are also displayed on the map, as shown in Figure 3. In Figure 3, the red terminal icon means that this terminal is in a state of failure. If there are some malfunctioning channels/terminals in the early warning area, the early warning system will give a suggestion of substitute dissemination means. Moreover, the early warning system can also be able to locate and search for emergency responders in the early warning area.

3) Performance evaluation after early warning information dissemination

The dissemination process of early warning information is monitored in real time, as shown in Figure 4. According to Figure 4, the user can clearly know the 


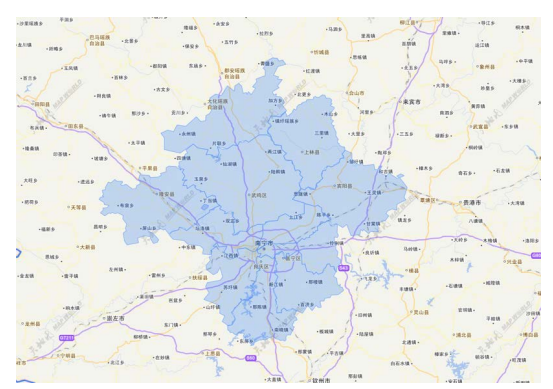

(a) Administrative area

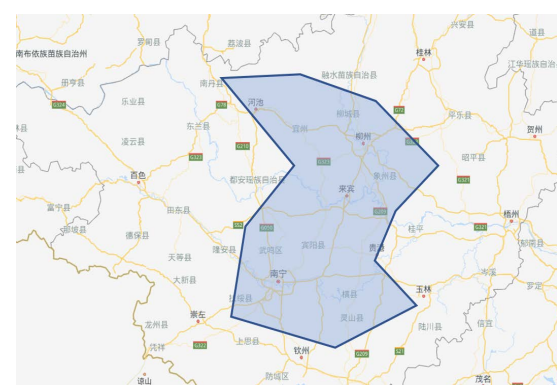

(b) Polygon area

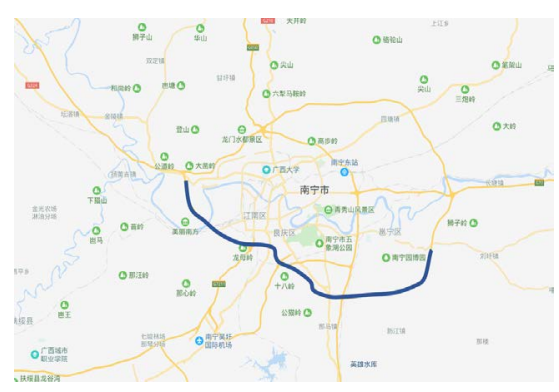

(c) Linear areas

Figure 2. The Type of the early warning area.

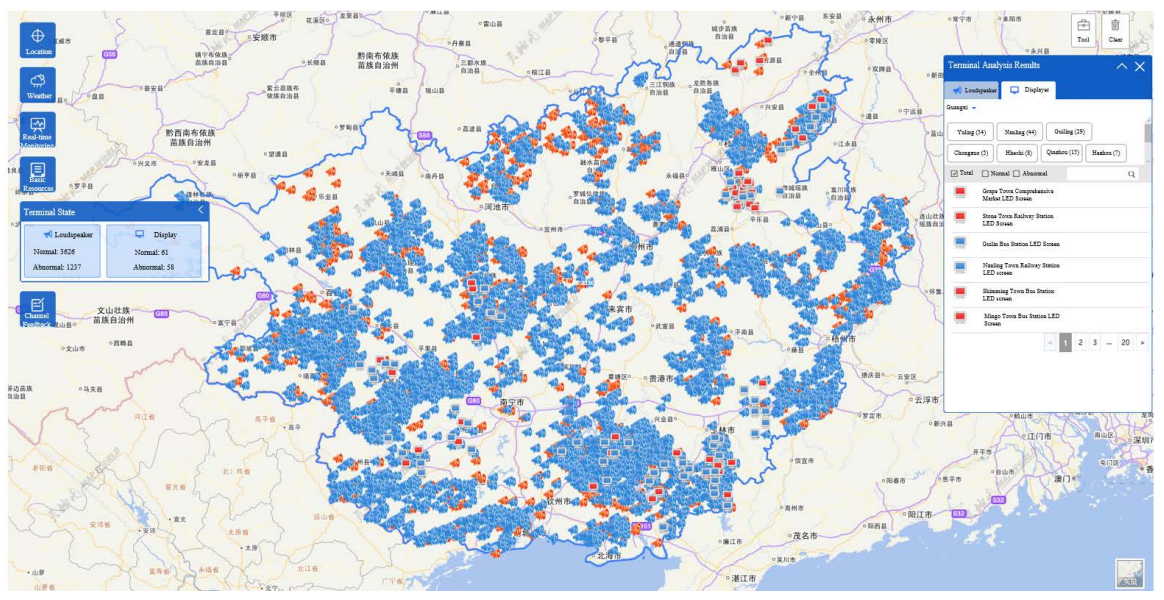

Figure 3. Dissemination terminal monitoring in the early warning areas.



Figure 4. Monitoring and display of the early warning dissemination process.

specific process of the early warning information dissemination. In addition, in order to ensure that every person in the affected area successfully and timely receives the early warning information, the system will evaluate the performance after the early warning information dissemination. And the evaluation content includes the spatial coverage rate, population coverage rate and timeliness of early warning information dissemination, which is shown in Figure 5. Based on the evaluation results, if some areas are not successfully disseminated, the system 


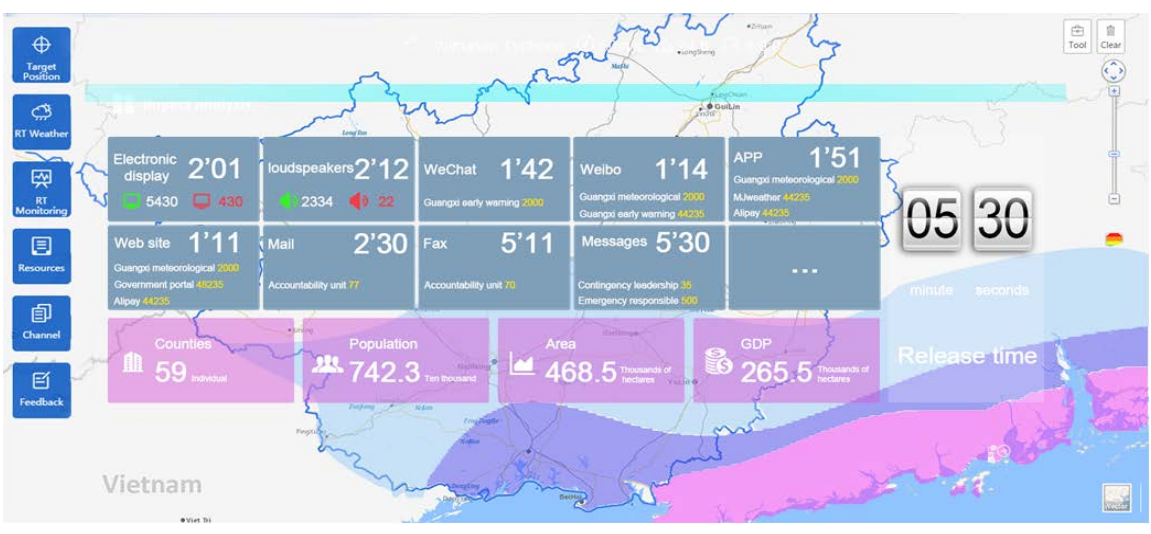

Figure 5. Performance evaluation after the early warning dissemination.

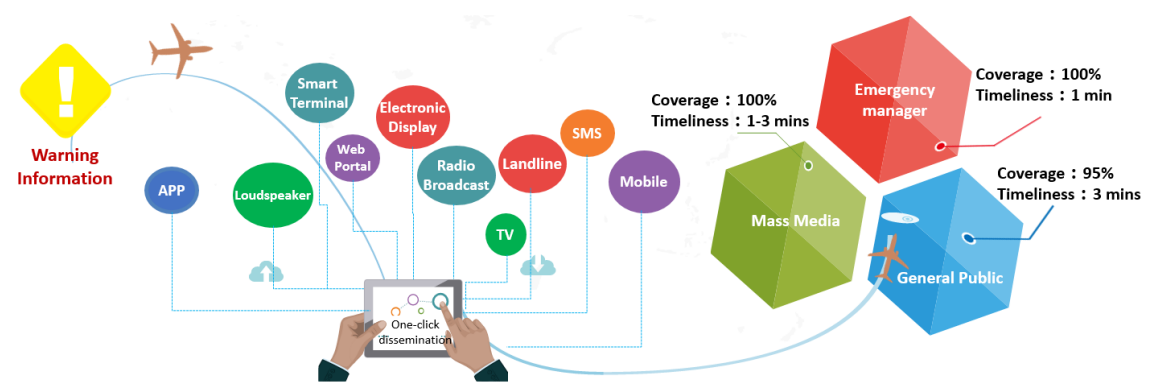

Figure 6. Performance of Guangxi Province early warning information dissemination system.

will re-disseminate the warning information to these areas, or select other channels to disseminate warning information to these areas.

\section{Application in Guangxi Early Warning System}

Guangxi early warning system for disasters and emergencies was implemented and launched in May 2017, which has a three-tiered architecture, i.e. provincial, municipal and county levels. The system supports 16 channels to disseminate early warning information, including TV, radio, website, SMS, fax, telephone, loudspeaker, email, mobile apps, electronic display, smart terminal, etc.

In order to improve the efficiency and accuracy of early warning information dissemination, the targeted dissemination technology has been built in this system. Since applying the targeted dissemination technology, the ability of early warning dissemination in Guangxi Province has been greatly improved. As shown in Figure 6, 100\% of the relevant emergency manager and social media can receive early warning information within 1 minute; and $95 \%$ of the public in the affected area can receive early warning information within 3 minutes. From May 20, 2017 to August 6, 2018, Guangxi early warning system disseminated 21,758 warning messages, including $118,694,046$ sent by SMS, 178,512 times via loudspeaker, and 45,342 times sent to display screens.

\section{Conclusion}

In this paper, application of incident chain model and targeted dissemination 
technology in early warning system has been investigated. Key features of this approach include flexible definition for early warning area, dissemination terminal performance monitoring and evaluation. In addition, the targeted dissemination technology was adopted in Guangxi early warning system, resulting in notably enhanced performance.

\section{Acknowledgements}

The authors are grateful for the support provided by the National Key Research and Development Program of China (Grant No. 2018YFC0808600), the National Natural Science Foundation of China (Grant No. 11802160), and the China Postdoctoral Science Foundation (Grant No. 2017M610091 and 2018T110106).

\section{Conflicts of Interest}

The authors declare no conflicts of interest regarding the publication of this paper.

\section{References}

[1] UNISDR (2015) Sendai Framework for Disaster Risk Reduction 2015-2030. 3rd United Nations World Conference on DRR, Sendai. https://doi.org/10.18356/02e5faa7-en

[2] Alparslan, E., Ince, F., Erkan, B., et al. (2008) A GIS Model for Settlement Suitability regarding Disaster Mitigation, a Case Study in Bolu Turkey. Engineering Geology, 96, 126-140.https://doi.org/10.1016/j.enggeo.2007.10.006

[3] Chen, P. and Cong, W.Q. (2006) Construction of the Geological Hazard Meteorological Warning System in Hunan Province Supported by GIS. Journal of Chengdu University of Technology, 33, 532-535. (In Chinese)

[4] Billa, L., Mansor, S. and Rodzi Mahmud, A. (2004) Spatial Information Technology in Flood Early Warning Systems: An Overview of Theory, Application and Latest Developments in Malaysia. Disaster Prevention and Management. An International Journal, 13, 356-363.https://doi.org/10.1108/09653560410568471

[5] Osanai, N., Shimizu, T., Kuramoto, K., et al. (2010) Japanese Early-Warning for Debris Flows and Slope Failures Using Rainfall Indices with Radial Basis Function Network. Landslides, 7, 325-338.https://doi.org/10.1007/s10346-010-0229-5

[6] Yuan, H.Y., Huang, Q.Y., Su, G.F., et al. (2007) Theory and Practice of Key Technologies Emergency Platform System. Chapter 3, Tsinghua University Press, Beijing. 